\title{
Circuit
}

Musiques contemporaines

\section{La musique au service de l'exposition Les temps inachevés de Patrick Bernatchez}

\section{Karine Bouchard}

Volume 26, numéro 3, 2016

URI : https://id.erudit.org/iderudit/1038521ar

DOI : https://doi.org/10.7202/1038521ar

Aller au sommaire du numéro

Éditeur(s)

Les Presses de l’Université de Montréal

ISSN

1183-1693 (imprimé)

1488-9692 (numérique)

Découvrir la revue

Citer ce document

Bouchard, K. (2016). La musique au service de l'exposition Les temps inachevés de Patrick Bernatchez. Circuit, 26(3), 90-94. https://doi.org/10.7202/1038521ar d'utilisation que vous pouvez consulter en ligne.

https://apropos.erudit.org/fr/usagers/politique-dutilisation/ 


\title{
La musique au service de l'exposition Les temps inachevés de Patrick Bernatchez
}

\author{
Karine Bouchard
}

Par la présentation de l'exposition Les temps inachevés de l'artiste d'arts visuels montréalais Patrick Bernatchez à l'automne 2015', le Musée d'art contemporain de Montréal (MACM) s'est engagé dans une voie qui a su accorder une importance considérable au son. Dans les espaces, un véritable jeu de résonance s'est établi entre les installations, les vidéos et parfois même les performances grâce à l'emprise sous-jacente du sonore qui rythmait le parcours, faisait écho sur l'architecture et orientait la perception des visiteurs quant aux éléments à regarder². Le discours de la commissaire Lesley Johnstone souligne justement ces rapports correspondants lorsqu'elle mentionne que «[1]'exposition met clairement en relief le lien inextricable entre l'oral et le visuel, le son et l'image, la musique et les arts plastiques ${ }^{3} »$.

Lartiste use de répétitions et de décalages afin de revisiter la temporalité, les Variations Goldberg et le piano. Véritables leitmotive, ces thèmes persistent et s'entremêlent dans l'espace d'exposition à travers différentes installations constamment teintées de formes multiples du sonore. Ils se déploient à travers deux cycles d'œuvres interdisciplinaires, Chrysalides (20062013) et Lost in Time (2009-2015), qui sont en constante mouvance - encore aujourd'hui -, justifiant conséquemment l'intitulé de l'exposition.

\section{Chrysalides $^{4}$}

Agissant comme une allégorie de l'édifice Fashion Plaza et de ses transformations de 2006 jusqu'à 2013, le cycle Chrysalides traduit matériellement et auditivement le concept de cet immeuble 5 . Le visiteur qui entre dans la première salle se trouve face à une installation colossale, Fashion Plaza Nights, l'une des créations majeures du cycle. Celle-ci se compose de fil industriel qui se déroule de 104 gigantesques bobines de manière à recouvrir un haut-parleur effectuant une lente rotation sur lui-même (figure 1). Pour accompagner ce mouvement, une musique plutôt mélancolique, diffusée de l'enceinte, rythme la mécanique et telle une trame sonore, crée une narration abstraite qui donne sens à la morphologie changeante des bobines et à ce qu'elles représentent, soit la lente modification de la fonction initiale du Fashion Plaza à Montréal ${ }^{6}$. Mais cette source sonore devient littéralement étouffée par l'accumulation de fils au fur et à mesure de la progression de l'exposition, émettant un son toujours plus sourd à chaque visite. Si l'impression première qui en ressort est l'évanouissement du son dû à une matérialité trop présente, il en est cependant tout autrement. En effet, la structure musicale s'inscrit de fait aux fondements mêmes de l'œuvre, puisque la vitesse de rotation des bobines de l'installation est contrôlée 
par des partitions programmées qui sont le résultat de 12 compositions pour deux pianos de l'artiste, dont la source de création réside dans son travail photographique antérieur ${ }^{7}$.

FIGURE 1 Patrick Bernatchez, Fashion Plaza Nights, 20072013. Installation: partitions, enregistrement sonore transféré sur support numérique, fil, supports, plateforme tournante, haut-parleurs, photographie; dimensions variables. Photo : Richard-Max Tremblay.

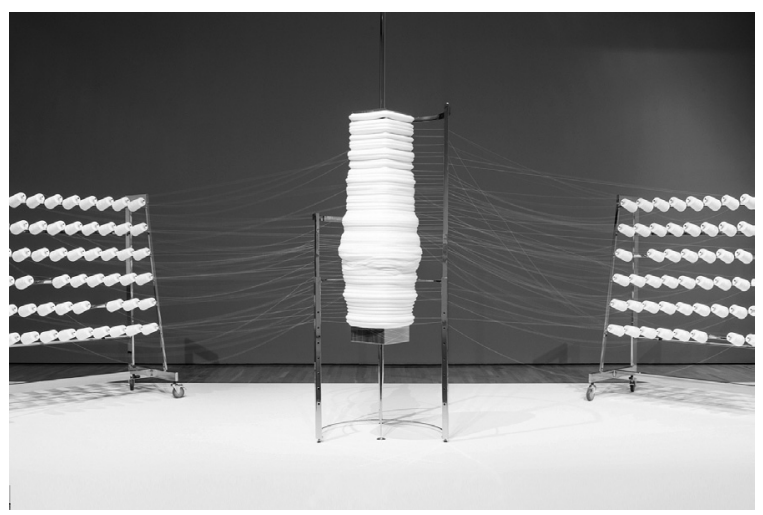

Chaque mois pendant un an, Bernatchez a fixé sur image les façades du Fashion Plaza en captant les variations de lumière qui transparaissaient à ce moment des fenêtres et qui traduisaient l'activité manufacturière des artisans. Il a ensuite transposé en une structure musicale cette luminosité et cette répétition des activités usinières. Concrètement, chaque variation de lumière est devenue une nuance (pianissimo, forte) ou un mode (majeur ou mineur), des tons et demi-tons désignant quant à eux la distance entre les étages du bâtiment. Dans l'espace d'exposition, les photographies sont présentées en clin d'œil à la composition. L'artiste propose ainsi un double décalage du visuel au sonore: de la photographie à la transcription musicale, à son interprétation par des instruments. C'est la transformation du paysage visuel qui permet, ici, l'élaboration de la musique.

\section{Lost in Time T $^{8}$}

Des projections à canal unique ainsi que des vinyles 33 tours sur des tables tournantes scandent la seconde partie du parcours occupée par le cycle Lost in Time. Le visiteur déambule d'abord à travers les installations de la série Piano orbital pour en faire l'expérience, tantôt en écoutant dans un casque une réinterprétation des pièces de Ravel, Hoyt, Lekeu, Bach ou Debussy, tantôt en entendant les sons se réverbérer sur les cimaises du musée. Exploitant également de multiples niveaux de signification, Lost in Time présente des enregistrements sur vinyle qui sont le résultat de partitions ayant été retournées quatre fois à $90^{\circ}$ et dont les nouvelles notes issues du procédé ont été retranscrites pour être ensuite jouées. Dans l'espace d'exposition, ces phénomènes de permutation qui traversent les pièces musicales rejoignent le film $180^{\circ}$ qui complète cette série. Le visiteur peut y observer les images de ce court-métrage qui dévoile, après un long travelling, un pianiste et son instrument inversés, en suspension dans l'espace. Sur l'écran un musicien interprète dans cette position une variation de la Sonate pour piano $n^{\circ} 4$ (date inconnue) de Guillaume Lekeu dans une salle de concert vide. De toute évidence, au milieu de ces correspondances, un procédé métonymique semble agir là où l'image des mains du pianiste évoque les différentes pièces musicales à l'écoute.

Le visiteur remarque déjà dans cette partie du parcours des bribes de la série d'œuvres Goldberg Experienced qui constitue tout un pan des recherches de l'artiste et qui s'intègre au cycle Lost in Time. Les traces de la performance qui a donné le ton à cette série, Goldberg Experienced.o1 Berlin Session (2010-2011), ont été récupérées pour les besoins de l'exposition. Lors de la performance originale, le pianiste David Kaplan a réinterprété les Variations Goldberg (c. 1740) de Jean-Sébastien Bach sur un piano à queue préparé. Pendant toute la durée de la prestation, le musicien ajoutait ou enlevait pour 
chaque variation des objets apposés sur les cordes de l'instrument, soit des matières choisies qui rappelaient celles utilisées pour la constitution de natures mortes hollandaises. Une fois transposé dans la salle d'exposition, ce processus performatif se manifeste par des images sur écran en stopmotion qui montrent le flétrissement des différents éléments, des fruits et des fleurs. Ce véritable memento mori est accompagné d'une station d'écoute qui permet de revivre dans son ensemble l'expérience de la performance.

\section{Goldberg Experienced ${ }^{9}$}

Nous l'avons mentionné, l'artiste a développé toute une production inspirée des Variations Goldberg ${ }^{10}$. Bernatchez expérimente l'œuvre du grand maître à travers différents médiums en variant chaque fois le contenu musical où les composantes se font écho et débordent même des salles d'exposition, dans une visée dialogique.

FIGURE 2 Patrick Bernatchez, $77 K, 1^{e r}, 2^{e}, 3^{e}$ mouvement (Goldberg Experienced.03), 2012. Installation: film de $16 \mathrm{~mm}$, 8 minutes en boucle, tournedisques, 8 vinyles 33 tours, son; dimensions variables. Photo: Patrick Bernatchez.

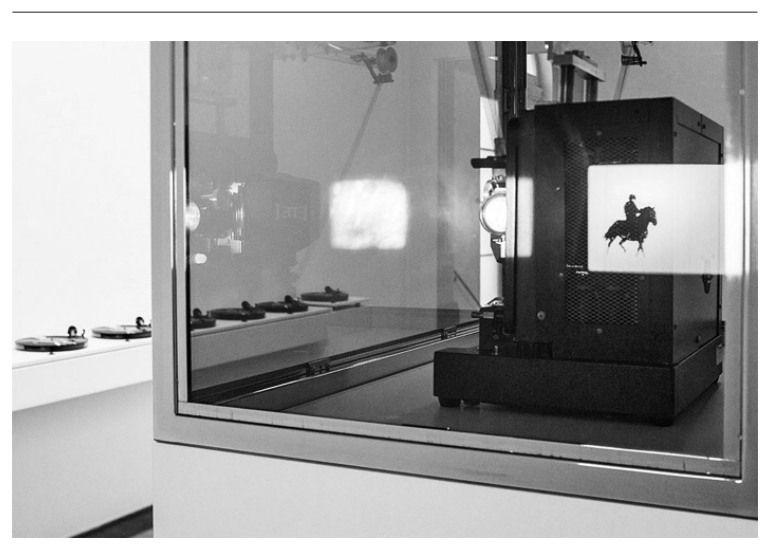

Déjà, dans la rotonde du MACM, à l'extérieur même des salles prévues à cet effet, le visiteur accède à l'expo- d'une version modifiée des Variations Goldberg diffusée grâce à huit haut-parleurs spatialisant le lieu ${ }^{11}$. Il faut toutefois poursuivre le parcours de l'exposition à l'étage pour voir la source sonore de cet enregistrement. Celle-ci apparaît dans l'installation $77 \mathrm{~K}, 1^{e r}$, $2^{e}, 3^{e}$ mouvement (Goldberg Experienced.o3) qui se compose de huit disques vinyles préparés de l'enregistrement des Variations interprétées par Glenn Gould en $1981^{12}$. Ces disques rejouent simultanément des fragments de l'œuvre: des modifications apportées à chaque vinyle usent les sillons, ce qui engendre une répétition en boucle de certains passages musicaux spécifiques, altère le phrasé et la durée de l'enregistrement initial de manière à déployer des versions singulières et décalées. L'ensemble crée alors une musique nouvelle. Autrement dit, le résultat se traduit par une démultiplication de la musique de Bach et de la performance de Gould au profit d'une ouvre devenue minimaliste, complexe et rythmiquement instable qui rappelle plutôt les expérimentations de Philip Glass ou de Steve Reich ${ }^{13}$. Un film sur pellicule en $16 \mathrm{~mm}$, qui est également présenté dans cette salle, semble être accompagné de cette musique. Et si ces sons donnent un rythme plus accéléré à l'image presque statique, ils renvoient surtout à l'iconographie du cavalier que l'on aperçoit à l'écran, lequel apparaît non seulement dans cette partie du parcours, mais également dans tout l'espace d'exposition de façon sporadique ${ }^{14}$.

Pour ajouter davantage et compléter cette intrication sonore entourant les Variations Goldberg, la composition issue de $77 \mathrm{~K}, 1^{e r}$, $2^{e}$, $3^{e}$ mouvement (Goldberg Experienced.03) que nous venons d'analyser a été transposée sur partition et jouée en concert au MACM comme finale de l'événement ${ }^{15}$. L'enregistrement s'est alors animé pour offrir un nouveau rapport visuel à l'œuvre: les corps des pianistes interprètes se sont substitués aux technologies, soit aux disques vinyles et haut-parleurs. 
Pour des informations audiovisuelles supplémen-

Lors de sa déambulation dans le parcours, le visiteur fait l'expérience, sans ordre prédéterminé, de différents espaces visuels, sonores et temporels. Concrètement, il écoute et observe la récurrence des leitmotive et leurs nombreuses permutations par des enregistrements sur disque vinyle et sur pellicule, ou encore par la modification de ces disques et de l'enregistrement de ces modifications, sinon lors du concert. À ces expériences s'ajoute son regard posé tantôt sur les images des photographies ou des courts-métrages, tantôt sur les disques-objets de la salle d'exposition qui renvoient à ceux des haut-parleurs de la rotonde. Les sons de ces lieux se répondent, lesquels dévoilent une image mnémonique de la performance musicale passée. En somme, un véritable vortex englobe divers moments d'écoute et d'observation des sons, des sonorités et des éléments visibles; ils deviennent interchangeables dans l'imaginaire du visiteur susceptible de recréer sa propre narration de l'exposition.

Les musiques déconstruites, réinterprétées et diffusées donnent une cohérence à l'ensemble ou en modifient le sens. Elles divulguent également la matière, unifiant ou décalant les espaces de création, ceux de l'exposition, d'une manière contrapuntique et polyphonique. Il semblerait ainsi que cette exposition de Patrick Bernatchez soit susceptible de donner raison à certains points de la pensée de Jean-Luc Nancy lorsque celui-ci mentionne que si la forme est « d'emblée pensée ou saisie dans l'ordre visuel », le sonore, lui, l'«emporte». Sans la dissoudre, il l'élargit, lui donne «une ampleur, une épaisseur et une vibration ou une ondulation ${ }^{16}{ }^{\prime}$. Lartiste joue de ces rapports parfois différentiels, parfois presque connectés. Il engage ainsi, dans Les temps inachevés, une relecture constante des œuvres par les glissements, les écarts, les brouillages ou les correspondances qui sont le plus souvent issus ou engendrés par les propriétés du sonore. taires, nous référons le lecteur au microsite de l'exposition du МАСм : <www.macm.org/patrickbernatchez/ lost-in-time/?dir=r $>$ (consulté le 16 septembre 2016).

1. La pratique multidisciplinaire de l'artiste montréalais Patrick Bernatchez s'élabore de manière à ce que chaque œuvre renvoie à un tout. La résurgence de motifs associés au temps, entre autres, fait correspondre les productions entre elles pour former des cycles. Le son et la musique ponctuent les créations et s'immiscent à travers différents médiums dont la vidéo, le film et l'installation. Bernatchez a exposé au Musée national des beaux-arts du Québec ainsi qu'à l'étranger à la galerie West (La Haye, Hollande), au Fresnoy - Studio national des arts contemporains (Tourcoing, France), au Mass MOCA (Massachusetts, États-Unis). Pour des informations plus détaillées, nous référons le lecteur au site web de l'artiste:

$<$ www.patrickbernatchez.com> (consulté le 16 septembre 2016).

2. L'exposition est une collaboration entre le Casino Luxembourg et le MACM. D’abord présentée au Luxembourg en septembre 2014 , l'exposition s'est ensuite tenue à Montréal du 17 octobre 2015 au 10 janvier 2016. Entre-temps, elle a occupé le Centre Argos (Bruxelles, Belgique) du 26 avril au 28 juin 2015 et la galerie West du 5 septembre au 3 octobre 2015. L'exposition s'est finalement déplacée à la galerie Power Plant (Toronto, Ontario) du 30 janvier au 15 mai 2016.

3. Lesley Johnstone (dir.) (2015), Patrick Bernatchez: les temps inachevés, Luxembourg, Casino Luxembourg, p. 4.

4. L'ensemble de ce cycle inclut également des courts-métrages disposés sporadiquement dans les salles suivantes. Parmi les projections, notons la trilogie des Chrysalides en $35 \mathrm{~mm}$, soit I Feel Cold Today, Chrysalide et 13, Chrysalide Empereur. Bien que toutes les œuvres du cycle méritent une attention particulière, nous avons dû, pour les besoins de cet article, sélectionner certaines d'entre elles qui illustrent précisément le rapport du son dans l'espace visuel du musée et qui favorisent les réflexions sur le sujet.

5. Le Fashion Plaza était un lieu montréalais qui abritait plusieurs fabricants de vêtements avant de subir un embourgeoisement qui a mené à l'expulsion des artisans.

6. L'importance de la musique de l'installation fait écho à celle de la trame sonore des films du même cycle mentionnés précédemment. Elle a été méticuleusement pensée par l'artiste pour en accompagner la narrativité

7. L'artiste a été témoin de tous les changements du lieu au fil des années, puisque son atelier se situait à même le Fashion Plaza.

8. Le cycle comprend plus de 20 œuvres elles-mêmes regroupées en différentes séries.

9. En 2012, la revue Circuit, musiques contemporaines a consacré un numéro (vol. 22, $n^{\circ} 2$ ) à Glenn Gould et la création. Dans l'enquête qu'a menée Flavia Gervasi sur l'influence de Gould auprès des 
compositeurs, interprètes et réalisateurs, Louis Dufort témoigne de sa collaboration avec Marie Chouinard qui l'a menée à la réalisation de l'œuvre Body Remix - Goldberg Variations à partir des Variations Goldberg interprétées par Gould (p. 52-53).

10. Les Variations Goldberg ont été une influence considérable pour plusieurs artistes et compositeurs, dont Karlheinz Essl qui introduit des sons électroniques dans la musique originale. <www.essl.at/ works/goldbergwerk.html> (consulté le 16 septembre 2016).

11. La pièce intitulée Goldberg Experienced.04 (GEO433RPM) d'une quarantaine de minutes était diffusée en boucle pendant toute la durée de l'exposition.

12. Des adhésifs apposés sur les sillons des disques ainsi que des égratignures font en sorte que l'aiguille de la table tournante grave à chaque fois les mêmes sillons.

13. Cette installation s'est animée dans le cadre des nocturnes du MACM grâce à un DJ qui interprétait la pièce. Goldberg Experienced.03.77 live a été présentée le 13 novembre 2015.
14. La musique fait également écho au long-métrage intitulé Lost in Time mettant en scène ce cavalier dont la trame sonore revisite une fois de plus l'œuvre de Bach. Le film est diffusé sur écran géant dans une salle isolée du reste de l'exposition. Il est en quelque sorte l'aboutissement des recherches de l'artiste sur la temporalité inachevée. Précisons que la trame sonore a été réalisée en collaboration avec le musicien Murcof.

15 Présentée le dimanche 10 janvier 2016 dans la salle Beverley Webster Rolph du MACM, la performance Goldberg Experienced.04 (GEO433ROM) a été réalisée en collaboration avec le compositeur et chef d'orchestre Patrice Coulombe. Lors de la prestation, sept pianistes du Conservatoire de musique jouaient chacun sur un piano droit sous la direction de la musicienne Marybelle Frappier. Les pianos étant alignés, chaque musicien interprétait sa partie de la pièce musicale simultanément avec les autres. Une première version (GEO433RPM) avait précédemment été réalisée au Luxembourg.

16. Jean-Luc Nancy (2002), À l'écoute, Paris, Galilée, p. 14-15.

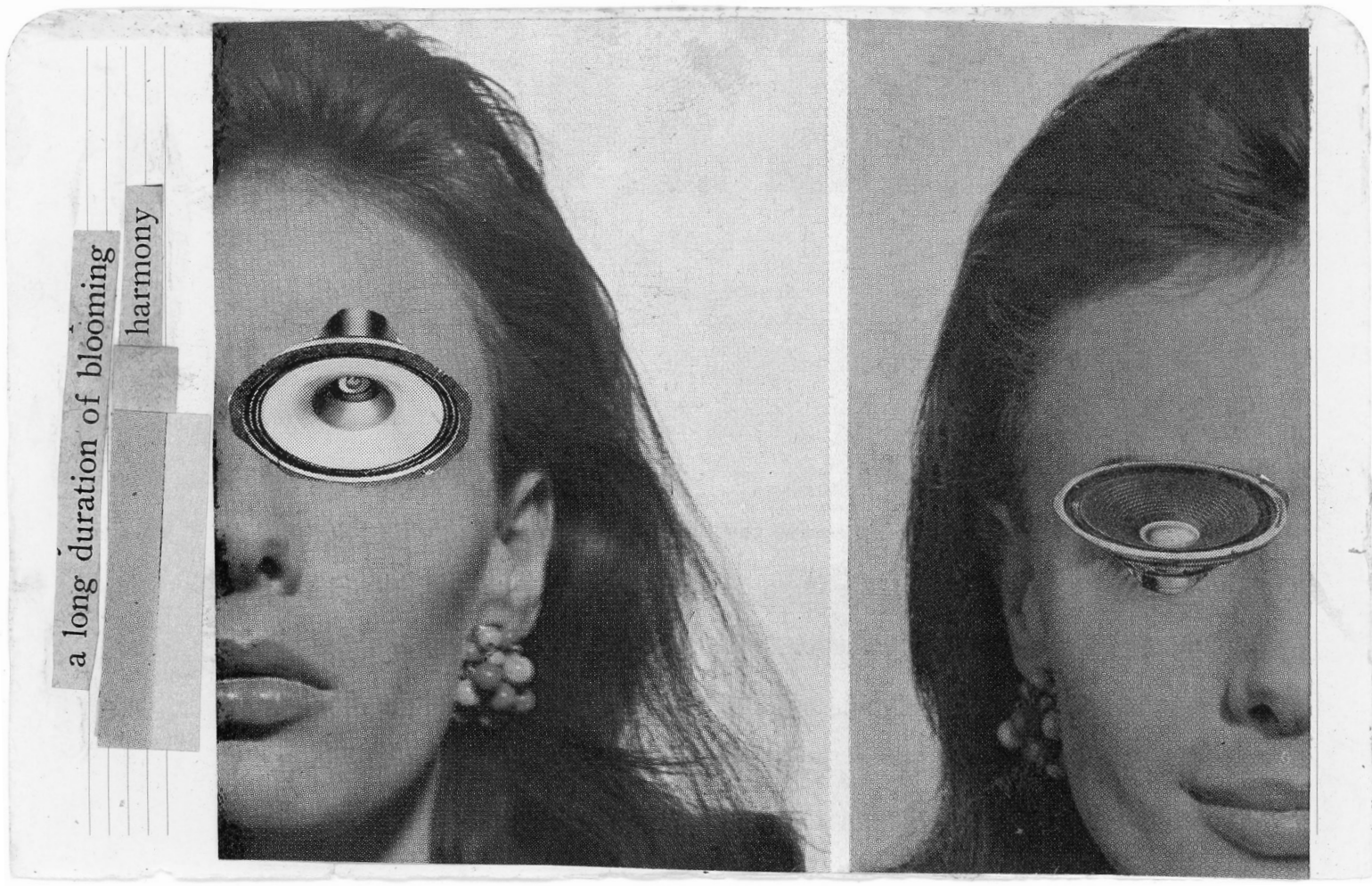

Steve Roden, Collage Scores, 2010. Collage sur papier à musique. 